\title{
Isometries of five-branes and T-duality
}

\author{
Edvard T. Musaev ${ }^{1,2, *}$ \\ ${ }^{1}$ Moscow Institute of Physics and Technology, Institutskii per. 9, Dolgoprudny, 141700, Russia \\ ${ }^{2}$ Kazan Federal University, Institute of Physics, Kremlevskaya 16a, 420111, Kazan, Russia
}

\begin{abstract}
T-duality chain starting from the NS5-brane is known to generate exotic backgrounds. These have been described as a DFT background whose orientation in the doubled space defines specific 10-dimensional solutions. The focus of this letter is at isometries of the corresponding background, their relation to the NS five-brane charge and the section constraint of DFT. This letter is based on the talk presented by the author at the conference QUARKS' 18.
\end{abstract}

\section{Branes and U-duality}

Analysis of dynamics of open string ends leads to the conclusion that spectrum of string theory contains Dp-branes in addition to the fundamental string F1 with tension proportional to $g_{s}^{-1}$ while for the string one has $T_{F 1} \sim g_{s}^{0}$ [1]. On the supergravity side these manifest themselves as black-brane solutions sourcing non-trivial flux of Ramond-Ramond fields $F_{(p+1)}=d C_{(p)}$ [2]. At the moment it is understood that spectrum of extended objects of string theory is infinitely rich and contains objects with $T \sim g_{s}^{-a}$ with $a$ not bound from above [3, 4]. However, only a subset of this infinite spectrum can be realized as branes of co-dimension up to zero in 10 dimensions (see e.g. [5, 6]).

To start with one considers the magnetic dual of the fundamental string, the NS5-brane, which interacts with the 6-form potential $B_{(6)}$ and has tension $T_{N S 5} \sim g_{s}^{-2}$. Compactifying the full 10D theory on a $\mathbb{T}^{7}$ and wrapping all the world-volume directions of the NS5-brane one ends up with a state of the 3D half-maximal supergravity whose mass is proportional to

$$
M\left[5_{2}^{0}\right] \sim \frac{R_{1} R_{2} R_{3} R_{4} R_{5}}{g_{s}^{2} l_{s}^{6}} .
$$

Here the notations in brackets descent from classification of massive states of the halfmaximal supergravity $b_{n}^{c}$, where $b$ and $c$ stand for the number of radii on which the mass depends linearly and quadratically respectively, and $n$ denotes the power of $g_{s}$ in the denominator [3, 4].

A state acquires non-zero $c$ when T-duality on directions absent in the mass formula is performed. For NS5-brane this corresponds to T-dualising along transverse directions, and leads to the Kaluza-Klein monopole (KK5) at the first step. And to non-geometric Qand R-branes when T-dualising further [4, 7, 8]. Fascinating is that while the NS5-brane magneticaly interacts with the 2-form gauge field $B_{(2)}$ via the 3 -form flux $H_{a b c}$, the KKmonopole magnetically interacts with gravitons via the geometric flux $f_{a b}{ }^{c}$. For this reason

*e-mail: musaev.et@phystech.edu 
KK5 can be understood as the magnetic dual of the graviton in the same sense as the NS5brane is the magnetic dual of F1. The corresponding 3D state $5_{2}^{1}$ will have the mass

$$
M\left[5_{2}^{1}\right] \sim \frac{R_{1} R_{2} R_{3} R_{4} R_{5} R_{6}^{2}}{g_{s}^{2} l_{s}^{8}},
$$

where $R_{i}$ denote radii of the compactified dimensions and the radius squared corresponds to the Taub-NUT direction (the special circle). Performing T-duality further one recovers the following chain

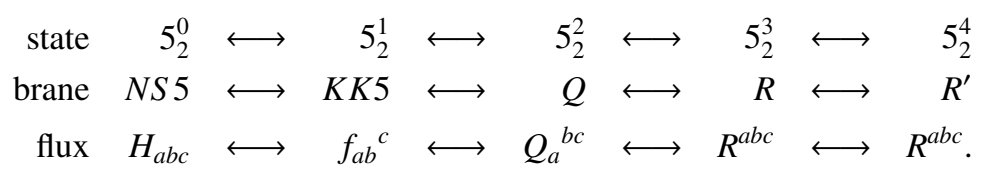

Here one faces an issue raised and resolved in [9], which is tightly related to double field theory techniques. To start with one notices, that to perform T-duality in a transverse direction a background has to be smeared in this direction to provide an isometry. After dualisation this becomes a special Taub-NUT-like direction, and the total number of such directions is given by the number $c$. Hence, the issue is that due to the different number of isometries of the NS5-brane background and the KK5 monopole the latter does not have infinite throat. To cure this, one considers world-volume instanton corrections, which localize the smeared NS5-brane back into the full brane background. Special transverse isometry directions were shown to get localized in the corresponding dual space [10-12].

This has the most transparent explanation from the point of view of Double Field Theory (DFT), where the space-time is doubled to allow independent right-moving and left-moving harmonics on closed string $[13,14]$. The mass spectrum is then encoded in terms of the so-called generalized metric

$$
\mathcal{H}_{M N}=\left[\begin{array}{cc}
G_{m n}-B_{m}{ }^{k} B_{k n} & B_{n}{ }^{q} \\
B_{m}{ }^{p} & G^{p q}
\end{array}\right],
$$

where $G_{m n}$ and $B_{m n}$ is the 10D space-time metric and the Kalb-Ramond 2-form field [15]. The doubled space is parametrized by coordinates $X^{M}=\left(x^{m}, \tilde{x}_{m}\right)$, which unify the normal and dual coordinates.

Using this representation in $[16,17]$ it has been shown that the backgrounds sourced by the branes from the chain (3) are all different faces of a single object, the DFT monopole, which differ by isometry directions. In [18] this setup for isometries has been used to construct a single effective action for the DFT monopole. In this text we focus on the isometry directions, the corresponding Killing vectors and relation between these and the section constraint of DFT.

\section{Symmetries of NS five-branes}

The NS5-brane background has six isometry directions which correspond to world-volume of the brane. T-duality along these isometries does not change the background. To arrive at the KK5 monopole one has to smear the NS5 brane along one of the transverse directions to generate an isometry and T-dualize along it. After dualization this isometry corresponds to the Taub-NUT direction. Hence, the number of isometries for the Kaluza-Klein monopole differs from that for the NS5-brane. To perform the conventional T-duality further one must increase the number of isometries to arrive finally at the space-filling configuration of the R'-brane, the $5_{2}^{4}$-state. 
In [10] taking into account world-sheet instanton corrections was shown to localize the smeared NS5-brane back to the original one, and localize the KK5-monopole in the direction $\tilde{z}$ dual to the special isometry direction. In $[11,12]$ the same has been shown for the $5_{2}^{2}$ brane, whose background acquires dependence on two dual coordinates. Hence, one naturally arrives at description of brane backgrounds in terms of doubled geometry $[16,17,19]$. This suggests to always consider the full localized branes and to count isometries in the doubled transverse space. Since T-duality along the world-volume directions does not change the brane and hence is trivial, these direction are not doubled.

Knowing which isometries a brane has in the full doubled space appears to be crucial for identification of the object. Indeed, consider the NS5-brane background which depends on coordinates $\left\{x^{6}, x^{7}, x^{8}, x^{9}\right\}$ and does not depend on the corresponding duals $\left\{\tilde{x}_{6}, \tilde{x}_{7}, \tilde{x}_{8}, \tilde{x}_{9}\right\}$, thus having four isometries. For the localized KK5-monopole with the Taub-NUT direction along $x^{9}$ one finds dependence on $\left\{x^{6}, x^{7}, x^{8}, \tilde{x}_{9}\right\}$, while the rest four directions are again isometries. Continuously replacing coordinates by their duals one moves along the T-duality chain (3) and obtains exotic configurations.

In [18] the notion of isometry encoded in a set of (constant) generalized vectors $k_{a}^{M}$, labelled by $a=1,2,3,4$, was employed to construct a single effective action for the T-duality orbit (3). This approach suggests to understand the representatives of the orbit as different orientations of a single object. Directions singled out by the vectors $k_{a}^{M}$ will correspond to isometry directions of the corresponding background, in addition these define the brane charge. Let us focus on this in more details.

The NS-NS part of the effective action for the orbit takes the following form

$$
S_{D B I}^{N S}=\int d^{6} \xi e^{-2 d} \sqrt{\operatorname{det} h_{a b}} \times \sqrt{-\operatorname{det}\left[g_{\mu \nu} \partial_{\alpha} X^{\mu} \partial_{\beta} X^{v}+\mathcal{H}_{M N} \hat{D}_{\alpha} Y^{M} \hat{D}_{\beta} Y^{N}\right]},
$$

where the integration is performed along the 5+1-dimensional world-volume, $g_{\mu \nu}$ and $\mathcal{H}_{M N}$ are the metric and the generalized metric, $h_{a b}=k_{a}{ }^{M} k_{b}{ }^{N} \mathcal{H}_{M N}$ is the auxiliary matrix, and $X^{\mu}$ and $Y^{M}$ are the world-volume scalar fields. Upon coupling to the DFT action the former will correspond to coordinates along the world-volume and the latter - to the doubled transverse coordinates. To make use of the information about isometries the fields $Y^{M}(\xi)$ enter via the projected derivatives defined as

$$
\hat{D} Y^{M}=d Y^{M}-h^{a b} k_{a}{ }^{M} k_{b}{ }^{N} \mathcal{H}_{N K} d Y^{K} .
$$

Such defined projector removes half of the fields $Y^{M}(\xi)$ from the effective action upon a choice of the explicit form of the vectors $k_{a}^{M}$. To do so these are taken to satisfy the following constraints

$$
\begin{aligned}
\eta_{M N} k_{a}{ }^{M} k_{b}{ }^{N} & =0, \\
\epsilon^{a b c d} k_{a}{ }^{M} k_{b}{ }^{N} k_{c}{ }^{K} k_{d}{ }^{L} & =Q^{M N K L} \neq 0 .
\end{aligned}
$$

The first line is the analogue of the section constraint of DFT and ensures that no more than half of the total set of vectors $k_{a}^{M}$ is non-zero. In principle $k_{a}^{M}=0$ also satisfies the first line, but that leads to keeping all the fields $Y^{M}(\xi)$ in the action and thus to breaking of the DFT's section constraint. To avoid this the second line above has been introduced, which tells that the brane should have non-vanishing charge $Q^{M N K L}$, and ensures that no less than half of the vectors should be non-zero. Together, these constraints keep exactly half non-zero vectors, which correspond to precisely four isometry directions of the background.

Using such defined charge, interaction of the DFT-monopole with gauge fields can be written in a (linearized) T-duality covariant form

$$
S_{W Z}^{l i n}=\int d^{6} \xi Q^{M N K L} D_{(6) M N K L}=\int d^{6} \xi \epsilon^{a b c d} k_{a}{ }^{M} k_{b}{ }^{N} k_{c}{ }^{K} k_{d}{ }^{L} D_{(6) M N K L} .
$$


Here $D_{(6) M N K L}$ is the 6-form gauge potential containing gauge fields interacting with the branes of $(3)$ (see $[6,20]$ for more details on that). To see explicitly how solutions of the constraint (7) reproduce the expected results for different branes one simply lists all possible solutions and splits them into sets not related by a $G L(4)$ rotation. Let us start with the NS5 brane which corresponds to

$$
k_{a}^{M}=\left(0_{a}{ }^{m} ; \tilde{k}_{a m}\right),
$$

where $m=6,7,8,9$ label the transverse directions. The (linear part of the) Wess-Zumino action then becomes

$$
\begin{aligned}
S_{W Z}^{l i n, N S 5} & =\int d^{6} \xi \epsilon^{a b c d} \tilde{k}_{a m} \tilde{k}_{b n} \tilde{k}_{c k} \tilde{k}_{d l} D_{(6)}{ }^{m n k l}=\operatorname{det}\left[\tilde{k}_{a m}\right] \int d^{6} \xi D_{(6)}{ }^{m n k l} \epsilon_{m n k l} \\
& =\mu_{N S 5} \int d^{6} \xi B_{(6)},
\end{aligned}
$$

where we introduce the magnetic dual of the Kalb-Ramond field $B_{(6)}$ and charge of the NS5brane $\mu_{N S 5}$ as

$$
\mu_{N S 5} B_{\mu_{1} \ldots \mu_{6}} d X^{\mu_{1}} \wedge \cdots \wedge d X^{\mu_{6}}=\operatorname{det}\left[\tilde{k}_{a m}\right] D_{(6)}{ }^{m n k l} \epsilon_{m n k l} .
$$

Hence, one concludes that the 5-brane invariant object oriented such as to have four isometries along dual directions reproduces the conventional NS5-brane. And the same is true for the full DBI action as shown in [18].

To switch to the (localized) Kaluza-Klein monopole one replaces one of the co-vectors $\tilde{k}_{a m}$ by a vector $k_{a}^{m}$ to get

$$
\begin{aligned}
k_{1}^{M} & =\left(k_{1}^{m} ; 0_{1 m}\right), \\
k_{\hat{a}}{ }^{M} & =\left(0_{\hat{a}}^{m}, \tilde{k}_{\hat{a} m}\right),
\end{aligned}
$$

where $\hat{a}=2,3,4$. Note that due to the first line of (7), which should be true for all $a$ and $b$, $\tilde{k}_{\hat{a} m}$ and $k_{1}{ }^{m}$ cannot have non-zero components for the same value of $m$. Taking all this into account one writes for the WZ term

$$
\begin{aligned}
S_{W Z}^{l i n, K K 5} & =\int d^{6} \xi \epsilon^{1 \hat{a} \hat{b} \hat{c}} k_{1}{ }^{m} \tilde{k}_{\hat{a} n} \tilde{k}_{\hat{b} k} \tilde{k}_{\hat{c} l} D_{(6) m}{ }^{n k l}=\int d^{6} \xi \epsilon^{1 \hat{a} \hat{b} \hat{c}} k_{1}{ }^{m} \tilde{k}_{\hat{a} n} \tilde{k}_{\hat{b} k} \tilde{k}_{\hat{c} l} \epsilon^{n k l p} D_{(6) m, p} \\
& =\operatorname{det}[(k, \tilde{k})] \int d^{6} \xi D_{(6) m, m}=\mu_{K K 5} \int d^{6} \xi \iota_{k_{1}} A_{(7,1)},
\end{aligned}
$$

where in the second line we used the fact that $p$ must be equal to $m$ and defined a $4 \times 4$ matrix

$$
(k, \tilde{k}):=\left[\begin{array}{c}
k_{1}^{m} \\
\tilde{k}_{\hat{a} m}
\end{array}\right] .
$$

The mixed symmetry gauge potential $A_{(7,1)}$ is defined via its components as $A_{\mu_{1} \ldots \mu_{7}, v}$ where $v$ must be equal to one of $\mu$ 's. Taking Hodge star of its field strength one recovers the geometric flux $f_{\mu \nu}{ }^{\rho}$. This potential enters the Wess-Zumino action above via interior product with the vector $k_{1}^{m}$

$$
\mu_{K K 5} \iota_{k_{1}} A_{(7,1)}=\mu_{K K 5} A_{\mu_{1} \ldots \mu_{6} m, m} d X^{\mu_{1}} \wedge \cdots \wedge d X^{\mu_{6}}=\operatorname{det}[(k, \tilde{k})] D_{(6) m, m}, \quad \text { (no sum). }
$$

The repeated index $m$ is there in order to keep the $G L(4)$ covariance of all expressions. For applications it is more convenient to switch to the adapted basis where only $k_{1}^{1} \neq 0$. In this case the non-vanishing components of the mixed-symmetry potential are $A_{\mu_{1} \ldots \mu_{6} 1,1}$.

In the conventional supergravity picture the repeated index of the potential $A_{(7,1)}$ always corresponds to 
- the Taub-NUT direction;

- the quadratic radius in the mass of the corresponding state (2).

Combining the above information one concludes that the repeated index, the Taub-NUT direction and the quadratic radius in the mass formula all correspond to having isometry in the conventional space and hence localization in the dual space.

Continuing the above procedure for other representatives of the orbit (3) one recovers the following mixed-symmetry potentials for the branes $5_{2}^{2}, 5_{2}^{3}$ and $5_{2}^{4}$

$$
\begin{aligned}
5_{2}^{2}: & \iota_{k_{1}} \iota_{k_{2}} B_{(8,2)} & \sim D_{(6) m n}{ }^{p q} \epsilon_{p q m n}, \\
5_{2}^{3}: & \iota_{k_{1}} \iota_{k_{2}} \iota_{k_{3}} B_{(9,3)} & \sim D_{(6) m n k}{ }^{p} \epsilon_{p m n k}, \\
5_{2}^{4}: & \iota_{k_{1}} \iota_{k_{2}} \iota_{k_{3}} \iota_{k_{4}} B_{(10,4)} & \sim D_{(6) m n k l} \epsilon_{m n k l},
\end{aligned}
$$

where sum is assumed only over repeated upper-lower indices.

As the final note for this section it is worth mentioning that the $(6+4)$ split of the coordinates is not necessary to construct the full T-duality covariant action, although convenient. Instead, one considers the full $(10+10)$-dimensional doubled space and considers ten vectors $k_{a}^{M}$, now $a=1 \ldots, 10$ and $M=1, . ., 20$. The full Wess-Zumino term can be written in analogy by making use of a 0 -form $D_{M N K L}$ and a charge $Q_{M_{1} \ldots M_{10}}$ constructed out of the ten vectors $k_{a}^{M}$. The world-volume necessarily becomes 6-dimensional. Next one notices that changing 6 out of 10 vectors does not changes anything in the action and goes back to its split-form to keep only relevant information.

\section{Orientation and section constraint}

Consistency of algebra of symmetries of DFT requires the so-called section constraint which says that all fields of the theory should depend only on half of the total set of coordinates. Similar feature appeared in the previous section to consistently define effective action for the NS five-brane T-duality orbit. Indeed, the vectors $k_{a}^{M}$ are always such that the derivatives $\hat{D}_{\alpha}$ project out half of the world-volume scalar fields $Y^{M}(\xi)$. Although the doubled coordinates $X^{M}$ and the scalar fields $Y^{M}(\xi)$ are related by coupling of the effective action and the field theoretical action, the constraints are of different nature. In this section we provide a detailed discussion of the relation.

Let us first note, that without reference to DFT the effective action $S_{D B I}+S_{W Z}$ does not contain information about section constraint. This is just a six-dimensional theory formulated in terms of 20 scalar fields $Y^{M}(\xi)$, which contains projections. Apparently, at this stage choosing different solutions to the constraint (7) for the vectors $k_{a}^{M}$ does not change the result. The projected forms of the action for these different choices are related simply by redefinitions $Y^{m}(\xi) \leftrightarrow Y_{m}(\xi)$ for a given $m$. This has an interesting consequence of invariance of the corresponding Rui-Takayanagi entropy for (the field theoretical limit of) Little String Theory [21]. This is simply a reflection of T-duality invariance of the effective action.

Things start to get changed when one refers to backgrounds, for which coupling to the DFT action is required. This can be organized as follows

$$
\begin{aligned}
& S_{\text {full }}= \\
& =\int d^{6} x \int d^{8} X \mathcal{L}_{D F T}\left[\mathcal{H}_{M N}, d\right]+\int d^{6} \xi \mathcal{L}_{\text {brane }}\left[Y^{M}\right] \\
& =\int d^{6} x \int d^{8} X\left[\mathcal{L}_{D F T}\left[\mathcal{H}_{M N}, d\right]+\int d^{6} \xi \mathcal{L}_{\text {brane }}\left[\partial_{\alpha} Y^{M}\right] \delta^{(6)}\left(x^{\mu}-X^{\mu}(\xi)\right) \delta^{(8)}\left(X^{M}-Y^{M}(\xi)\right)\right],
\end{aligned}
$$


where we are using the split-form of DFT with $x^{\mu}$ being the external coordinates. The first Dirac-delta stands for alignment of the branes' world-volume coordinates along the external (non-doubled) space, while the second takes care of the choice of the representative of the T-duality orbit. Next, one must agree on which subset of the coordinates $X^{M}=\left(x^{m}, \tilde{x}_{m}\right)$ is geometric and which is dual, i.e. which of these are used to measure distances in the usual space-time. It is convenient to set $x^{m}$ to be the geometric coordinates.

Now, let us start with a solution of (7) corresponding to, say, the NS5-brane, which reads

$$
k_{a}^{M}=\left(0, \tilde{k}_{a m}\right)
$$

Calculating the projected derivatives explicitly one obtains

$$
\hat{D}_{\alpha} Y^{m}=\partial_{\alpha} Y^{m}, \quad \hat{D}_{\alpha} Y_{m}=B_{m n} \partial_{\alpha} Y^{n}
$$

i.e. the fields $Y_{m}(\xi)$ are completely dropped from the action. Since the source term does not contain dynamical field $Y_{m}(\xi)$ it is natural to expect that the resulting background will not depend on the corresponding coordinates $\tilde{x}_{m}$. Additionally, gauge fixing the fields $X^{\mu}(\xi)=$ $\delta_{\alpha}^{\mu} \xi^{\alpha}$ and $Y^{m}\left(x^{\alpha}\right)=0$ (on the brane) one obtains

$$
S_{\text {full }}^{N S 5}=\int d^{6} x \int d^{4} x^{m}\left[\operatorname{Vol}_{(0,4)} \mathcal{L}_{D F T}\left[\mathcal{H}_{M N}, d\right]+\mathcal{L}_{\text {brane }}\left[\partial_{\mu} Y^{m}\right] \delta^{(4)}\left(x^{m}\right)\right],
$$

which produces a solution localized in four transverse directions $x^{m}$. The volume $\operatorname{Vol}_{(0,4)}$ of the dual space appeared from integration over $\tilde{x}_{m}$, and appears to be a common prefactor for the DFT Lagrangian. Checking the explicit form of the Lagrangian $\mathcal{L}_{\text {brane }}$ for the projection in question one ensures that this solution is indeed the NS5-brane background (see [18] for detailed derivation).

Note, that of crucial importance for obtaining the NS5-brane background from the solution (18) was the convention about geometric and dual coordinates. To demonstrate this, consider the case of the Kaluza-Klein monopole, for which one has

$$
k_{1}^{M}=\left(k_{1}^{m} ; 0\right), \quad k_{\hat{a}}^{M}=\left(0 ; 0, k_{\hat{a} m}\right) .
$$

After all gauge fixings and integrations the corresponding full effective action in adapted coordinates becomes

$$
S_{\text {full }}^{K K 5}=\int d^{6} x \int d \tilde{x}_{1} d^{3} x^{i}\left[\operatorname{Vol}_{(1,3)} \mathcal{L}_{D F T}\left[\mathcal{H}_{M N}, d\right]+\mathcal{L}_{\text {brane }}\left[\partial_{\mu} Y^{i}, \partial_{\mu} \tilde{Y}_{1}\right] \delta\left(\tilde{x}_{1}\right) \delta^{(3)}\left(x^{i}\right)\right] .
$$

Now the projected derivatives leave only the fields $\left\{\tilde{Y}_{1}(\xi), Y^{i}(\xi)\right\}$ with $i=2,3,4$ and the corresponding coordinate $x^{1}$ gets integrated, since the background does not depend on it as for the conventional Kaluza-Klein monopole. The new feature coming from DFT here is dependence on $\tilde{x}_{1}$ reflected by the localization $\delta\left(\tilde{x}_{1}\right)$. Again, writing the Lagrangian $\mathcal{L}_{\text {brane }}$ explicitly one convinces oneself that this is indeed the action for the KK monopole.

The subtle and very important point here is, that the only difference between (22) and (20) is the convention about $x^{1}$ and $\tilde{x}_{1}$. For background fields this reflects in the fact that for the natural convention with $x^{1}$ being geometric we understand $\mathcal{H}_{11}=g_{11}+B_{1 m} g^{m n} B_{n 1}$, while for the opposite convention, when $x^{1}$ is non-geometric this will be $\mathcal{H}_{11}=g^{11}$. Apparently, these two are related by T-duality along $x^{1}$ as it should be for KK5 and NS5.

To conclude let us list the sets of constraints and their meaning in this context

- section condition: ensures closure of the DFT algebra, works only inside $\mathcal{L}_{D F T}$, i.e. for target space fields; 
- the constraint (7): removes half of the world-volume scalar fields from the effective action, needed to keep world-volume supersymmetry.

In addition, given the section constraint is satisfied and target space fields depend only on half of the coordinates, one has to agree which half of the full set of coordinates are geometric. Going through these steps allows to uniquely identify a brane background from the full effective action of [18].

\section{Acknowledgements}

The authors' attendance of the conference QUARKS'2018 was sponsored by the Russian state grant Goszadanie 3.9904.2017/8.9 and in part by the Alexander von Humboldt return fellowship.

\section{References}

[1] R.G. Leigh, Mod. Phys. Lett. A4, 2767 (1989)

[2] J. Polchinski, Phys.Rev.Lett. 75, 4724 (1995), hep-th/9510017

[3] N. Obers, B. Pioline, Phys.Rept. 318, 113 (1999), hep-th/9809039

[4] J. de Boer, M. Shigemori, Phys. Rept. 532, 65 (2013), 1209.6056

[5] A. Kleinschmidt, JHEP 10, 144 (2011), 1109. 2025

[6] E.A. Bergshoeff, F. Riccioni, JHEP 05, 131 (2011), 1102 . 0934

[7] J. Shelton, W. Taylor, B. Wecht, JHEP 0510, 085 (2005), hep-th/0508133

[8] B. Wecht, Class.Quant.Grav. 24, S773 (2007), 0708 . 3984

[9] D. Tong, JHEP 07, 013 (2002), hep-th/0204186

[10] J.A. Harvey, S. Jensen, JHEP 10, 028 (2005), hep-th/0507204

[11] T. Kimura, S. Sasaki, JHEP 08, 126 (2013), 1305 . 4439

[12] T. Kimura, S. Sasaki, K. Shiozawa, JHEP 07, 001 (2018), 1803. 11087

[13] A.A. Tseytlin, Phys.Lett. B242, 163 (1990)

[14] W. Siegel, Phys.Rev. D48, 2826 (1993), hep-th/9305073

[15] O. Hohm, C. Hull, B. Zwiebach, JHEP 1008, 008 (2010), 1006.4823

[16] I. Bakhmatov, A. Kleinschmidt, E.T. Musaev, JHEP 10, 076 (2016), 1607.05450

[17] D.S. Berman, F.J. Rudolph, JHEP 05, 015 (2015), 1409. 6314

[18] C.D.A. Blair, E.T. Musaev, JHEP 03, 111 (2018), 1712.01739

[19] S. Jensen, JHEP 1107, 088 (2011), 1106. 1174

[20] E.A. Bergshoeff, F. Riccioni, Phys. Lett. B704, 367 (2011), 1108. 5067

[21] A.A. Golubtsova, E.T. Musaev (2018), 1806.02297 